\title{
Comparación entre el bosque templado austral y el bosque tucumano- boliviano considerando géneros con especies de hábito arbóreo
}

\section{Comparison between the austral temperate forest and the tucumano-boliviano forest through genera with species of arboreal habits}

\author{
María Paula Quiroga
}

Laboratorio Ecotono, INIBIOMA-CONICET / Centro Regional Universitario Bariloche, Quintral 1250. Bariloche, Río Negro. Argentina.

pquiroga@crub.uncoma.edu.ar; emepequ@gmail.com

\begin{abstract}
RESUMEN
Las áreas fitogeográficas del bosque tucumano-boliviano (BTB) de Argentina y Bolivia y el bosque templado austral (BTA) de Argentina y Chile están separadas por la diagonal árida (aproximadamente $2.000 \mathrm{~km}$ entre bosques). El objetivo de este trabajo es conocer cuántos géneros de especies con hábito arbóreo se distribuyen dentro de los bosques templado austral y tucumano-boliviano. En este trabajo se analiza y compara los géneros con especies de hábito arbóreo distribuidos en cada bosque. Además se indica el elemento biogeográfico para cada género. Se detectaron 125 y 29 géneros, en el BTB y en el BTA respectivamente, y los restantes 13 estaban presentes en ambos bosques. Los 167 géneros totales se distribuyeron en ocho elementos biogeográficos. Los elementos más abundantes fueron el neotropical y pantropical, seguidos del austral y endémico del bosque templado. Los dos primeros fueron más frecuentes en el BTB, mientras que los dos últimos fueron en el BTA. Los géneros que son compartidos en ambos bosques correspondieron a los elementos austral, neotropical, pantropical y holártico. A partir de los géneros que están compartidos se postulan distintas hipótesis para explicar las disyunciones. Una hipótesis es que habrían tenido una distribución antigua amplia y que las condiciones geo-climáticas produjeron la disyunción. Otra hipótesis es que ocurrieron eventos de dispersión a larga distancia luego de la formación de la diagonal árida. Los géneros restringidos al BTA serían relictos de un origen antiguo en Gondwana y los géneros restringidos al BTB serían productos de numerosas diversificaciones relativamente locales.
\end{abstract}

Palabras clave: Bosque templado austral, bosque tucumano-boliviano, disyunciones arbóreas sudamericanas, géneros arbóreos, historia biogeográfica sudamericana.

\begin{abstract}
The fitogeographic areas from the tucumano-boliviano forest (BTB) from Argentina and Bolivia and the temperate austral forest (BTA) from Argentina and Chile are separated by de arid diagonal (approximately by 2,000 km between forests). The objective of this work is to know how many genera with species of arboreal habits are distributed in the temperal austral and tucumano-boliviano forests. In this work, the genera with species of arboreal habits distributed in each forest are analyzed and compared. In addition, the biogeographic element for each genus is shown. 125 and 29 genera were detected in BTB and in BTA respectively, and the remaining 13 genera were present in both forests. The total 167 genera were distributed in eight biogeographic elements. The most abundant elements were the neotropical and pantropical, followed by the austral and temperate forest endemic. The first ones were more frequent in the BTB, while the other two elements were more frequent in the BTA. The genera shared by both forests corresponded to the austral, neotropical, pantropical, and holartic elements. From these shared genera, different hypotheses to explain the disjunction are postulated. One hypothesis is that they may have distributed widely in ancient times and that the geoclimatic conditions caused the disjunction. Another hypothesis is that long distance dispersal events occurred after the formation of the arid diagonal. Restricted BTA genera would suggest relictual status of an ancient origin in Gondwanaland, while restricted BTB genera would be products of various relatively local diversifications.
\end{abstract}

KEYWORDS: Temperal austral forest, tucumano-boliviano forest, South America arboreal disjunction, arboreal genera, South America biogeographical history. 


\section{INTRODUCCIÓN}

Las distribuciones actuales de las familias, géneros y especies de plantas están mayormente asociadas a los eventos que ocurrieron a partir del Cretácico tardío hasta fines del Cenozoico. Durante la era Cenozoica la Tierra experimentó grandes cambios climáticos, desde un calentamiento generalizado con los polos libres de hielo en el período Paleógeno, cuando muchos géneros tropicales se originaron y diversificaron en el continente sudamericano (Jaramillo et al. 2006). Durante el Eoceno, el registro fósil indica una alta diversidad de especies de plantas (Barreda \& Palazzesi 2007, Iglesias et al. 2007, Wilf et al. 2003). Posteriormente, se registra un importante cambio climático dando lugar a intensos fríos en los polos, los que finalmente produjeron el comienzo de la glaciación de la Antártida (Birkenmajer et al. 2005). Así mismo, en Sudamérica se incrementó la aridez (Clarke 2006). En el Mioceno medio se establece la corriente circumpolar Antártica, la cual trae aparejados importantes cambios climáticos, especialmente la glaciación completa de la Antártida (Ramos \& Kay 1992, Ortiz-Jaureguizar \& Cladera 2006). También durante este período se levantan los Andes, afectando notablemente las condiciones climáticas del continente, especialmente a ambos lados de la cordillera y a lo largo de toda su extensión (Graham et al. 2001, Blisniuk et al. 2005, Garreaud et al. 2010). Este importante acontecimiento causó un progresivo incremento en la aridez (Houston \& Hartley 2003) a causa de la barrera que los Andes produce sobre los vientos (Garreaud 2009, Garreaud et al. 2010) y la reducción de la concentración de $\mathrm{CO}_{2}$ atmosférico (Willis \& McElwain 2002). Hacia finales del Neógeno comienzan las glaciaciones sobre los continentes (Zachos et al. 2001), que culminaron con las grandes oscilaciones climáticas del Cuaternario (Clapperton 1993). La distribución de las regiones biogeográficas de Sudamérica es el resultado de la interacción de plantas y animales con los diferentes paleoclimas que se sucedieron y con las fuerzas geológicas que moldearon la geografía del continente sudamericano, como por ejemplo los cambios en el nivel del mar (Romero 1986, Ortiz-Jaureguizar \& Cladera 2006), orogenias de cadenas montañosas (Ramos 2005), vulcanismo (Stern 2008) y glaciaciones (Clapperton 1993, Thomson et al. 2010, Glaser et al. 2008). Los cambios consecuentes sufridos por la flora de Sudamérica durante el Paleógeno y Neógeno han sido analizados en diversas áreas (OrtizJaureguizar \& Cladera 2006), tanto con datos fósiles (Wilf 2003, Gayó et al. 2005, Jaramillo et al. 2006, Barreda et al. 2007, Prámparo et al. 2007), como moleculares (Richardson et al. 2001, Pennington et al. 2005, Marquínez et al. 2009, Mathiasen \& Premoli 2010). Muchas familias y géneros de plantas se han originado o diversificado especialmente en el neotrópico (Richardson et al. 2001, Pennington et al. 2005, Marquínez et al. 2009) o en áreas relativamente modernas como los ambientes proporcionados por la cordillera de los Andes (Panero \& Funk 2008). Otros, con los sucesivos cambios climáticos y nuevas formaciones orogénicas, se han extinguido localmente (Barreda et al. 2007). Y otros han quedado disyuntos en Sudamérica a raíz de los eventos geológicos y climáticos ocurridos desde el Paleógeno (Villagrán \& Hinojosa 1997, Fiaschi \& Pirani 2009). Muchos de los géneros y especies disyuntas han quedado actualmente en estructuras boscosas como por ejemplo las yungas, la mata atlántica y bosque templado austral. Estos bosques presentan ciertas condiciones ambientales equivalentes, en parte por su geología montañosa, que le otorga condiciones climáticas favorables para el desarrollo de especies con características ecológicas similares, y que además están emparentadas taxonómicamente, posiblemente con una distribución geográfica más amplia en el pasado.

Dos grandes áreas boscosas se encuentran actualmente distanciadas por aproximadamente $2.000 \mathrm{~km}$. Estos bosques son el tucumano-boliviano (de aquí en adelante abreviado como BTB) y el templado austral (de aquí en adelante abreviado como BTA) (Fig. 1). El primero corresponde al extremo sur de la selva subtropical de montaña o Yungas. Como estructura florística las "Yungas" tienen una amplia distribución latitudinal en Sudamérica (desde Venezuela hasta los $28^{\circ}$ en Argentina). En este trabajo sólo se analiza el sector más austral de esta área fitogeográfica conocido como el bosque o selva tucumano-boliviano (Killeen et al. 1993, Cabrera 1994, Navarro \& Ferreira 2004). Esta corresponde a una formación de bosque semi-húmedo montano que se extiende desde los $18^{\circ}$ hasta los $28^{\circ} \mathrm{S}$ sobre la cordillera Oriental del centro-sur de Bolivia hasta el noroeste de Argentina, desde los $800 \mathrm{~m}$ en el fondo de los valles hasta más de 3.000 m s.n.m. (Fig. 1). Tanto en Argentina como en Bolivia, el BTB se organiza en pisos altitudinales con vegetación de características fisonómico-florísticas bien definidas (Navarro \& Ferreira 2004, Brown et al. 2001). Mientras que el BTA es una isla biogeográfica sobre el margen suroeste del sur de Sudamérica (Armesto et al. 1996), que se distribuye desde los $38^{\circ} \mathrm{S}$ hasta el Cabo de Hornos a los $55^{\circ}$ S (según Luebert \& Pliscoff 2006), abarcando desde las costas del océano Pacífico, en el centro-sur de Chile, hasta las laderas este de la cordillera de los Andes, y culmina por el extremo austral del continente (Fig. 1). En este bosque, la flora arbórea se distribuye entre el nivel del mar hasta aproximadamente los $1.500 \mathrm{~m}$ s.n.m. En ambos bosques, el límite máximo de elevación se encuentra en el extremo norte de las distribuciones, cuyo nivel superior varía acorde a la variación general de elevación observada a lo largo de toda la cordillera de los Andes. Estos dos bosques formaban una continuidad durante el Paleógeno (Barreda \& Palazzesi 2007), ya que durante el óptimo climático del Eoceno los registro fósiles indican que las floras tropicales persistieron en el centro de Chile (Gayó et al. 2005), y quedaron separados a partir de la extensión de la diagonal árida que 
forma los desiertos australes de Sudamérica (Fig. 1), desde el Neógeno (Solbrig 1978, Hinojosa \& Villagrán 1997, Ruggiero \& Ezcurra 2003, Mancini et al. 2005, Placzek et al. 2009). A partir de los eventos geo-climáticos que produjeron el aislamiento de estas dos áreas fitogeográficas, en este trabajo se analiza y compara cuáles son los géneros que presentan especies de hábito arbóreo que se encuentran actualmente distribuidos en alguno de estos dos bosques o en ambas áreas fitogeográficas, es decir que sean géneros "comunes" a ambos bosques.

Con el fin de revisar cuáles son los taxones actuales que nos permiten establecer las "similitudes o vínculos" florísticos entre dos bosques que habrían formado una estructura continua en el pasado, este trabajo tiene como objetivo conocer cuántos géneros de especies con hábito arbóreo se distribuyen entre el BTB y el BTA (Fig. 1), distinguiendo entre aquellos que sólo habitan uno de los bosques y los que están presentes en ambos bosques o son "comunes". La hipótesis general es que los eventos geoclimáticos del pasado, ocurridos a distintas latitudes, afectaron diferencialmente la flora de Sudamérica en la composición relativa de elementos biogeográficos a nivel de géneros de especies con hábito arbóreo. Se espera una mayor proporción de géneros asociados a climas tropicales en el BTB, mientras que en el BTA se espera que los géneros asociados a climas más fríos sean relativamente más abundantes. Esta predicción esta sustentada como consecuencia, principalmente, de procesos de especiación y diversificación en el neotrópico (Richardson et al. 2001) y procesos de extinción local de géneros tropicales en el bosque templado austral (Barreda \& Palazzesi 2007) durante la aridización y enfriamiento de comienzos del Neógeno. Por esta razón, se espera encontrar un número similar de géneros compartidos o comunes en relación al elemento biogeográfico al que pertenecen.

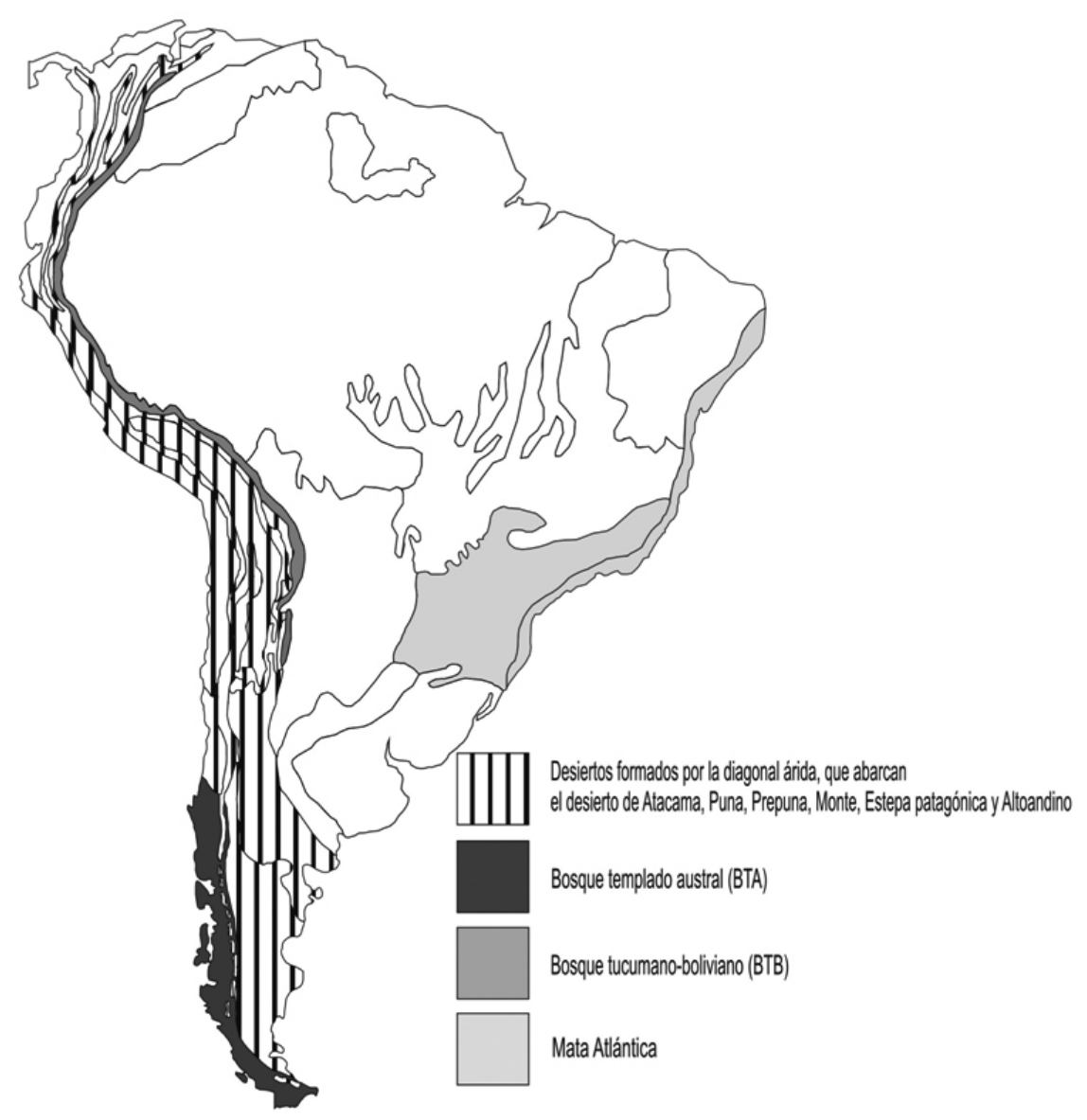

FIGURA 1: Regiones fitogeográficas de Sudamérica. (Según Cabrera y Willink 1973). Se indican en escala de gris el bosque templado austral (BTA), el bosque tucumano-boliviano (BTB) y la mata atlántica. Las regiones fitogeográficas que constituyen la diagonal árida se indican en rayado.

Figure 1: Phytogeographical regions of South America. (According to Cabrera and Willink 1973). The gray scale indicates the temperal austral forest (BTA), tucumano-boliviano forest (BTB) and the mata atlantica. The fitogeographic regions that comprise the arid diagonal are indicated in vertical lines. 
Bosque templado austral vs. bosque tucumano-boliviano: QUIROGA, M.P.

\section{MATERIALES Y MÉTODOS}

ÁREAS ESTUDIADAS

Las áreas estudiadas incluyen al bosque tucumanoboliviano y el bosque templado austral. El BTB comprende en esta comparación al centro y sur de Bolivia y noroeste de Argentina, abarcando desde los $18^{\circ} \mathrm{S}$ a los $28^{\circ} \mathrm{S}$. Representa el extremo austral de la selvas subtropicales de montaña o Yungas (Killeen et al. 1993, Cabrera 1994, Navarro \& Ferreira 2004). Esta zona es marcadamente diferente a los bosque húmedos del norte de Bolivia y de Perú, que continúan al norte de esta distribución (Navarro \& Ferreira 2004).

El BTA del suroeste del sur de Sudamérica se distribuye aproximadamente entre $\operatorname{los} 38^{\circ} \mathrm{S}$ y $55^{\circ} \mathrm{S}$ (el límite norte se ha tomado según la distribución del piso vegetacional de Nothofagus obliqua y Cryptocarya alba, según Luebert \& Pliscoff 2006), es considerado una isla biogeográfica por encontrarse aislado de otras masas boscosas (Armesto et al. 1996). El BTA tiene un origen antiguo (Mathiasen \& Premoli 2010), se han encontrado fósiles en la Antártida que se remontan al Cretácico superior (Poole et al. 2000, 2003) de especies leñosas similares a las especies que ocurren actualmente en la zona del bosque valdiviano (aproximadamente a los $40^{\circ} \mathrm{S}$ ).

El BTB y el BTA distan aproximadamente $2.000 \mathrm{~km} \mathrm{y}$ actualmente están separados por la diagonal árida (Fig. 1), formada por los desiertos de la Puna, Prepuna, Monte, Chaco Árido, Matorral Chileno y Estepa Patagónica (Cabrera \& Willink 1973, Mancini et al. 2005), donde las precipitaciones son generalmente menores a $500 \mathrm{~mm}$ anuales. La diagonal árida (Solbrig 1978, Mancini et al. 2005, Clarke 2006, Placzek et al. 2009), que mantiene florísticamente aisladas a estas dos áreas fitogeográficas, se habría originado en el norte con el desierto de Atacama, para el cual se tiene evidencia que ya hacia finales del Triásico sería una zona seca (Clarke 2006). Sin embargo, los procesos geoclimáticos ocurridos desde el Paleoceno hasta el Holoceno contribuyeron al desarrollo de la llamada diagonal árida (Graham et al. 2001, Houston \& Hartley 2003, Mancini et al. 2005, Barreda \& Palazzesi 2007, Garreaud 2009, Garreaud et al. 2010).

Para el presente trabajo se elaboró una lista de géneros de especies con hábito arbóreo de la flora del BTB y del BTA, los géneros de especies que se distribuyen en ambos bosques se indican como comunes. La lista de géneros se estructuró a partir de las siguientes publicaciones: Killeen et al. (1993), Dimitri et al. (1997), Aizen \& Ezcurra (1998), Navarro \& Ferreira (2004), Ezcurra \& Brion (2005), Donoso (2006), Luebert \& Pliscoff (2006) y Zuloaga et al. (2008) según datos publicados en www2.darwin.edu.ar. Además se utilizaron los siguientes sitios de internet: www.gbif. net, www.efloras.org y www.ipni.org para corroborar los nombres.

Según la distribución geográfica actual de los géneros
(Arroyo et al. 1996, Aizen \& Ezcurra 1998, Mabberley 2008), se asignó a cada género una de las siguientes categorías de distribución (Ulloa-Ulloa \& Møller-Jorgensen 1993, Arroyo et al. 1996, Aizen \& Ezcurra 1998) con el fin de conocer cómo son las proporciones de géneros en relación a su posible origen biogeográfico: austral (AU) (presente en bosques templados de América del Sur y otros continentes del hemisferio sur, como por ejemplo Australia y Nueva Zelanda), andino (AD) (en los Andes), cosmopolita (C) (en la mayoría de los continentes), endémico (E) (restringido al bosque templado del sur de Sudamérica), holártico o boreal $(\mathrm{H})$ (la mayor diversidad de especies están en áreas templadas del hemisferio norte, pero a veces se extienden $\mathrm{a}$ áreas montañosas frías y elevadas como los Andes en el hemisferio sur), neotropical $(\mathrm{N})$ (en áreas tropicales y subtropicales de América) y pantropical (P) (en los trópicos de América, Asia y/o África). Se supone que estas categorías están relacionadas con la posible área de origen de un taxón dado (e. g. Simpson \& Todzia 1990, Armesto \& Vidiela 1993, Ulloa-Ulloa \& Møller-Jorgensen 1993, Arroyo et al. 1996, Aizen \& Ezcurra 1998, Moreira-Muñoz 2007). En general, una distribución austral está considerada como posible evidencia de un origen remoto en el continente de Gondwana, y una distribución holártica como evidencia de origen en el hemisferio norte.

ANÁLISIS DE LOS DATOS

Se analizaron las proporciones de las distintas categorías de distribución geográfica de los géneros con especies de hábito arbóreo del BTB y del BTA mediante una prueba G de independencia (Sokal \& Rohlf 1981) para determinar si se mantienen las mismas proporciones entre categorías en las dos áreas boscosas.

\section{RESULTADOS}

Se identificaron un total de 167 géneros de especies con hábito arbóreo, distribuyéndose 125 géneros en el BTB, 29 géneros en el BTA, excluyentes respectivamente del otro bosque, y 13 géneros que se distribuyen en ambos bosques y por lo tanto se los denomina comunes (Tabla I, Material Suplementario). Los elementos con mayor número de géneros en el BTB fueron los de distribución neotropical y luego los de distribución pantropical, mientras que los géneros endémicos del BTA y australes dominan en el BTA (Tabla I). Las diferencias en las proporciones de géneros de diferentes elementos son estadísticamente significativas entre los dos bosques $(\mathrm{G}=130, \mathrm{p}<0.0001)$.

Los géneros del elemento endémico del BTA pertenecen a familias que se distribuyen en el hemisferio sur (e.g. Aextoxicaceae (Aextoxicon), Gomortegaceae (Gomortega), Proteaceae (Embothrium), Monimiaceae (Laureliopsis) y Podocarpaceae (Saxegothaea)) o que presentan una 
distribución más global y son pantropicales (e.g. Myrtaceae (Amomyrtus, Tepualia, Legrandia y Luma) y Cupressaceae (Austrocedrus, Fitzroya y Pilgerodendron)) (datos para familias no mostrados).

Los géneros compartidos, o que se encuentran en ambos bosques comparados, son cinco géneros del elemento neotropical (Lithrea, Schinus, Dasyphyllum, Citronella, Blepharocalyx), cuatro géneros del elemento austral
(Weinmannia, Crinodendron, Podocarpus, Prumnopitys), tres del elemento pantropical (Maytenus, Acacia, Persea) y uno del elemento holártico (Salix) (Tabla I, Material Suplementario). De estos géneros comunes, algunos presentan especies con amplias distribuciones, como es el caso de Salix humboldtiana Willd. y Maytenus boaria Molina las que se encuentran distribuidas en ambos bosques (datos para especies no mostrados).

TABLA I: Número de géneros según su distribución geográfica, que se encuentran en el sur del bosque tucumano-boliviano (BTB) y/o en el bosque templado austral (BTA) de América del Sur. AD: Andino, AU: Austral, C: Cosmopolita, E: Endémico, H: Holártico, N: Neotropical, P: Pantropical, S/D: sin datos.

TABLE I: Number of genera according to their geographical distribution, found in the southern tucumano-boliviano forest (BTB) and / or in the temperate austral forest (BTA) from southern South America. AD: Andean, AU: Austral, C: Cosmopolitan, E: Endemic, H: Holartic, N: Neotropical, P: Pantropical, S / D: no data.

\begin{tabular}{lcccc}
\hline Elementos & BTB & Comunes & BTA & Total por elemento \\
\hline AD & 3 & 0 & 0 & 3 \\
AU & 0 & 4 & 15 & 19 \\
C & 3 & 0 & 0 & 3 \\
E & 0 & 0 & 12 & 12 \\
H & 7 & 1 & 0 & 8 \\
N & 81 & 5 & 1 & 87 \\
P & 29 & 3 & 1 & 33 \\
S/D & 2 & 0 & 0 & 2 \\
\hline Total por bosque & 125 & 13 & 29 & 167 \\
\hline
\end{tabular}

\section{DISCUSIÓN}

La comparación de géneros con especies de hábito arbóreo, entre dos áreas boscosas de Sudamérica, mostró un alto porcentaje de géneros neotropicales y pantropicales en el BTB y la predominancia de géneros de los elementos australes y endémicos del bosque templado en el BTA. En el BTA se destaca un elevado número de endemismos (Arroyo et al. 1996) a nivel de género. En el BTB no se han detectado endemismos a este nivel, no obstante la mayor cantidad de endemismos en el bosque tucumanoboliviano ocurre a nivel de especies, en relación a las especies endémicas del BTA (Arroyo et al. 1996, Brown \& Malizia 2006). Se ha postulado que las especies endémicas del BTB serían producto de los aislamientos originados por los cambios climáticos del Cuaternario, suficientemente efectivos como para permitir especiación (Morales et al. 1995). En este sentido posiblemente el sector sur de Yungas, es decir la alta cuenca del río Bermejo en Argentina y Bolivia, habría funcionado como refugio de biodiversidad durante el Pleistoceno (Brown et al. 2001, Quiroga \& Premoli 2007). Los géneros del elemento endémico del BTA pertenecen a familias del hemisferio sur (cinco géneros) y a familias pantropicales (siete géneros) (datos no mostrados). La condición de endemismo para familias y géneros del hemisferio sur en el BTA sería producto de bajas tasas de extinción durante el Paleógeno en el sector sur de Sudamérica (Markgraf et al. 1995). Sin embargo, los géneros de familias pantropicales podrían ser relictos de linajes con más especies, y mayor extensión en el Paleógeno, que fueron reducidos por el enfriamiento y aridización del Neogeno (Barreda \& Palazzesi 2007). Este es el caso de los registros fósiles publicados en los trabajos de Iglesias et al. (2007) y Wilf et al. (2003, 2005). Posiblemente la diferencia entre extinción y permanencia entre estos dos grupos (familias del hemisferio sur y pantropicales) este asociada a las características de tolerancias ecológicas.

La estabilidad climática del Paleógeno habría permitido una amplia distribución de ciertos géneros, tanto de elementos australes en el BTB como de elementos neotropicales en el 
BTA (Hinojosa y Villagrán 1997). Asimismo, los registros fósiles de la flora indican una alta diversidad de plantas en todo el continente sudamericano en las primeras etapas del Eoceno (Jaramillo et al. 2006), incluido el norte de Patagonia (Hinojosa \& Villagrán 1997 según registros de la latitud $42^{\circ}$ S, Wilf et al. 2003, 2005). Las actuales disyunciones de géneros, es decir los que son comunes en ambos bosques, pueden ser reflejo de una distribución antigua amplia, y su permanencia o no, en ambos bosques, luego de la formación de la diagonal árida, podría ser simplemente por la conservación de nicho, que es la tendencia de las especies ancestrales para conservar las características ecológicas (Wiens \& Graham 2005). Sin embargo, también podría postularse que las restricciones ecológicas para el cruce de la diagonal árida han sido similares para géneros de distintos elementos biogeográficos. No obstante, en el caso de los elementos holárticos, los cuales ingresaron a Sudamérica durante el Plioceno (Simpson 1983, Simpson \& Todzia 1990), habrían utilizado la cordillera de los Andes para llegar a altas latitudes, por lo que dispusieron de menos tiempo para poder distribuirse ampliamente. La existencia de un mayor número de géneros holárticos en el BTB que en el BTA sugiere que la barrera de la diagonal árida filtró este elemento templado en su avance hacia el sur, o bien que el tiempo transcurrido no fue suficiente para sortear las barreras geológicas y climáticas y permitir que estos géneros se distribuyan a mayores latitudes. Por lo tanto, la cantidad de géneros holárticos a altas latitudes es menor que a bajas latitudes. Los géneros de especies de hábito arbóreo que tienen distribuciones disyuntas en el continente son de elementos de origen neotropical (Blepharocalyx, Citronella, Dasyphyllum, Lithrea, Schinus), austral (Crinodendron, Podocarpus, Prumnopitys, Weinmannia), pantropical (Acacia, Maytenus, Persea) y holártico (Salix). Además, ocurren disyunciones de géneros distribuidos en el BTA y en otros sectores boscosos de Sudamérica (e.g. la mata atlántica, otros sectores de yungas sensu lato), como los géneros Araucaria, Coriaria, Drimys, Lomatia, Myrceugenia, Podocarpus, Prumnopitys, Rhaphithamnus y Ugni, entre otros y con distintas formas de vida (Arroyo et al. 1996, Villagrán \& Hinojosa 1997, DeForest Safford 2007, Fiaschi \& Pirani 2009). Los géneros que presentan distribución disyunta en una o más estructuras boscosas sudamericanas podrían evidenciar eventos de vicarianza, o de dispersión temprana, como la que se propone para el género Podocarpus, el cual habría utilizado las incipientes montañas de la actual cordillera de los Andes para migrar desde el sur al norte de Sudamérica durante ciertos períodos del Eoceno (Van der Hammen \& Hooghiemstra 2001). La importancia actual de ciertas similitudes ecológicas se puede corroborar en los géneros que presentan las disyunciones. Este es el caso de Podocarpus, donde distintas especies se desarrollan en el BTB (P. parlatorei), en la mata atlántica $(P$. lamberti) y el BTA ( $P$. saligna y $P$. nubigena). De todas las especies mencionadas, únicamente $P$. nubigena pertenece a la sección Australis, las restantes a la sección Capitulatis. La evidencia filogenética molecular resuelta por Quiroga (2009) indica mayor grado de similitud entre las especies de la sección Capitulatis que entre especies próximas geográficamente. De esta manera las proximidades filogenéticas entre especies emparentadas pueden ser reflejo de áreas con características ecológicas similares que fueron continuas en el pasado y que actualmente están separadas.

Para los géneros disyuntos es interesante poner a prueba hipótesis que tengan una base ecológica para explicar posibles migraciones a larga distancia. Estas podrían postularse sobre la base de si son géneros con diferente, o similar, síndrome de dispersión de frutos. Así, puede asumirse que la selección de un mecanismo en particular de larga distancia podría ser evidencia de dispersión y la existencia de una variedad de mecanismos de corta distancia (viento, mecánico, aves, etc.) de vicarianza. En cuanto al análisis de hipótesis para estudiar los géneros disyuntos, todavía no se cuenta con filogeografías ni filogenias de taxones con estas características, con la intención de explicar cómo ha sido la historia que dejó a estos géneros (y especies) disyuntos en el continente. A pesar de ello, Marquínez et al. (2009) estudiaron el género Drimys con el fin de conocer las edades moleculares de las divergencias de este género. Las disyunciones entre las especies de Drimys tropicales y templadas sudamericanos, presentan evidencia molecular de diversificación que varía entre los 13 y los $16 \mathrm{Ma}$, cuando comenzaba a levantarse la cordillera de los Andes. Tanto las hipótesis ecológicas como las moleculares pueden ser analizadas en complementariedad con datos del registro fósil para resolver si las disyunciones son producto de asilamientos o migración.

Las afinidades florísticas entre el BTB y el BTA, analizadas mediante géneros de especies con hábito arbóreo permiten afirmar que a lo largo de la historia las especies, con estrechas relaciones filogenéticas y por lo tanto con una historia de vida común en un ancestro, resolvieron de manera independiente, es decir cada una según su ubicación geográfica y tolerancias ecológicas, los efectos de los eventos geológicos y climáticos ocurridos durante el Paleógeno, Neógeno y Cuaternario. Si bien este trabajo está limitado a una única forma de vida, los estudios filogeográficos y filogenéticos de géneros y especies disyuntas en Sudamérica, incluyendo otras formas de vida y taxones con otras características ecológicas, permitirán postular hipótesis sobre la historia biogeográfica de Sudamérica y proponer estrategias para la protección de posibles corredores entre áreas aisladas. Asimismo, los datos moleculares permitirán determinar si lo que actualmente denominamos como especies disyuntas realmente lo son, o ya han ocurrido suficientes mutaciones, o cambios moleculares, como para detectar genéticamente nuevas especies, favorecidas por restricciones al flujo génico. Además, la utilización de 
diferentes herramientas (genéticas, fósiles, reproductivas, morfológicas) para analizar las disyunciones permitirá postular distintas hipótesis para los diferentes grupos, y determinar si han sido fragmentaciones en la distribución o migraciones posteriores a las barreras que dejaron separadas dos áreas con características ecológicas similares. De esta manera, las predicciones presentadas en este trabajo para los géneros comunes pueden pasar a ser futuras hipótesis a ser analizadas.

No hay duda que acontecimientos como el cierre del Istmo de Panamá, el levantamiento de los Andes y los cambios climáticos del Cuaternario desempeñaron un papel importante en la configuración de patrones de biodiversidad (Bush \& Hooghiemstra 2005, Villagrán \& Hinojosa 1997). Por lo tanto, los efectos de los cambios paleogeográficos del Mioceno y Plioceno están relacionados con la formación de puentes y obstáculos a lo largo de América del Sur, modificando así la migración y los patrones de aislamiento, y favoreciendo la dispersión y vicarianza (Coyne \& Orr 2004).

\section{AGRADECIMIENTOS}

A Cecilia Ezcurra, Andrea Premoli, Cintia Souto, Paula Mathiasen y Nancy González por su valioso apoyo y sus sugerencias en beneficio de este trabajo. A los revisores anónimos por sus comentarios y sugerencias que han enriquecido este trabajo. La autora es becaria posdoctoral del CONICET.

\section{BIBLIOGRAFÍA}

Aizen, M. \& C. Ezcurra. 1998. High incidence of plant-animal mutualism in the woody flora of the temperate forest of southern South America: biogeographical origin and present ecological significance. Ecología Austral 8: 217236.

Armesto, J. \& P. Vidiela. 1993. Plant life-forms and biogeographic relations of the flora of Lagunillas in the fog-free Pacific Coastal Desert. Annals of the Missouri Botanical Garden 80: 499-511.

Armesto, J., P. Lobos \& M. Arroyo. 1996. Bosques templados del sur de Chile y Argentina: una isla biogeográfica. En: J.J. Armesto, C. Villagrán \& M.T.K. Arroyo (eds.), Ecología de los bosques nativos de Chile. pp. 23-27. Editorial Universitaria. Chile.

Arroyo, M., L. Cavieres, A. Peñalosa, M. Riveros \& A. Faggi. 1996. Relaciones fitogeográficas y patrones regionales de riqueza de especies en la flora del bosque lluvioso templado de Sudamérica. En: J.J. Armesto, C. Villagrán \& M.T.K. Arroyo (eds.), Ecología de los bosques nativos de Chile. pp. 71-92. Editorial Universitaria. Chile.

Barreda, V. \& L. Palazzesi. 2007. Patagonian vegetation turnovers during the Paleogene-Early Neogene: Origin of aridadapted floras. The Botanical Review 73: 31-50.
Barreda, V., L.M. Anzótegui, A.R. Prieto, P. Aceñolaza, M.M.A. Bianchi, M. Borromei, M. Brea, M. Caccavari, G.A. Cuadrado, S. Garralla, S. Grill, G.R. Guerstein, A.I. Lutz, M.V. Mancini, L.R. Mautino, E.G. Ottone, M.E. Quattrocchio, E.J. Romero, M.C. Zamaloa \& A. Zucol. 2007. Diversificación y cambios de las angiospermas durante el Neógeno en Argentina. Ameghiniana 50 aniversario: 173-191.

Blisniuk, P.M., L.A. Stern, C.P. Chamberlain, B. Idelman \& K. Zeitler. 2005. Climatic and ecologic changes during Miocene surface uplift in the southern Patagonian Andes. Earth Planetary Science Letter. 230: 125-142.

Brown, A. \& L. Malizia 2007. Lista comentada de los árboles de las Yungas de Argentina. En: A.D. Brown, M.G. Moritán, B.N. Ventura, N.I. Hilgert \& L.R. Malicia (eds.), Finca San Andrés. Un espacio de cambios ambientales y sociales en el Alto Bermejo. Ediciones del Subtrópico. Fundación ProYungas. 325 pp.

Brown, A.D., H.R. Grau, L.R. Malizia \& A. Grau. 2001. Argentina. En: M. Kappelle \& A. Brown (eds.). Bosques nublados del Neotrópico. pp. 621-659. Inbio. Costa Rica.

Birkenmajer, K., A. Gazdzicki, K.P. Krajewski, A. Przybycin, A. Solecki, A. Tatur \& H.I. Yoon. 2005. First Cenozoic glaciers in West Antarctica. Polish Polar Research 26: 312.

Bush, M.B. \& H. Hooghiemstra. 2005. Tropical biotic responses to climate change. In: T.E. Lovejoy \& L.Hannah (eds.). Climate Change and Biodiversity. pp. 125-137. Yale University Press, New Haven.

Cabrera, A.L. 1994. Regiones fitogeográficas argentinas, 2nd ed. ACME, Buenos Aires. 85 pp.

Cabrera, A.L. \& A. Willink. 1973. Biogeografía de América Latina. OEA, Washington. $120 \mathrm{pp}$.

Clapperton, C. 1993. Quaternary geology and geomorphology of South America. Elsevier. 769 pp.

Clarke, J. 2006. Antiquity of aridity in the Chilean Atacama Desert. Geomorphology 73: 101-114.

Coyne, J.A. \& H.A. OrR. 2004. Speciation. Sinauer, Sunderland. $545 \mathrm{pp}$.

DeForest SAFford, H. 2007. Brazilian paramos IV. Phytogeography of the campos de altitude. Journal of Biogeography 34: 1701-1722.

Dimitri, M., R. Leonardis, \& J. Santos Biloni. 1997. El nuevo libro del árbol. Tomo I. Especies forestales de la Argentina occidental. El Ateneo, Buenos Aires, Argentina. 120 pp.

Donoso, C. 2006. Las especies arbóreas de los bosques templados de Chile y Argentina. Autoecología. Marisa Cuneo Ediciones. Valdivia. Chile. 678 pp.

Ezcurra, C. \& C. Brion. 2005. Plantas del Nahuel Huapi, catálogo de la flora vascular del Parque Nacional Nahuel Huapi, Argentina. Universidad Nacional del Comahue y Red Latinoamericana de Botánica, San Carlos de Bariloche. Argentina. 70 pp.

Fiaschi, P. \& J.R. Pirani. 2009. Review of plant biogeographic studies in Brazil. Journal of Systematic and Evolution 47: 477-496.

Garreaud, R.D. 2009. The Andes climate and weather. Advances in Geosciences 22: 3-11.

Garreaud, R.D., A. Molina \& M. Farias. 2010. Andean uplift, ocean cooling and Atacama hyperaridity: A climate 
Bosque templado austral vs. bosque tucumano-boliviano: QuIROGA, M.P.

modeling perspective. Earth and Planetary Science Letters 292: 39-50.

Gayó, E., L.F. HinOJosa \& C. Villagrán. 2005. On the persistence of Tropical Paleofloras in central Chile during the Early Eocene. Review of Palaeobotany and Palynology 137: 4150.

Glasser, N.F., K.N. Jansson, S. Harrison \& J. Kleman. 2008. The glacial geomorphology and Pleistocene history of South America between $38^{\circ} \mathrm{S}$ and $56^{\circ} \mathrm{S}$. Quaternary Science Reviews 27: 365-390.

Graham, A., K.M. Gregory-Wodzicki \& K.L. Wright. 2001. Studies in Neotropical Paleobotany. XV. A Mio-Pliocene palynoflora from the Eastern Cordillera, Bolivia: implications for the uplift history of the Central Andes. American Journal of Botany 88: 1545-1557.

Hinojosa, L. \& C. Villagrán. 1997. Historia de los bosques del sur de Sudamérica, I: antecedentes paleobotánicos, geológicos y climáticos del Terciario del cono sur de América. Revista Chilena de Historia Natural 70: 225-239.

Houston, J. \& A.J. Hartley. 2003. The central Andean west-slope rainshadow and its potential contribution to the origin of hyper-aridity in the Atacama Desert. International Journal of Climatology 23: 1453-1464.

Iglesias, A., P. Wilf, K.R. Jonson, A.B. Zamuner, N. R. Cuneo \& S.D. Mateos. 2007. A Paleocene lowland macroflora from Patagonia reveals significantly greater richness than North America analogs. Geology 35: 947-950.

Jaramillo, C., M. Rueda \& G. Mora. 2006. Cenozoic plant diversity in the neotropics. Science 311: 1893-1896.

Killeen, T., E. García \& S. Beck. 1993. Guía de árboles de Bolivia. Herbario Nacional de Bolivia. Missouri Botanical Garden. $958 \mathrm{pp}$.

Luebert, F. \& P. Pliscoff. 2006. Sinopsis bioclimática y vegetacional de Chile. Editorial Universitaria. Santiago de Chile. 316 pp.

Mabberley, D. 2008. Mabberley's Plant-book. A Portable Dictionary of Plants, their Classifications, and Uses. 3edt. Edition University of Washington Botanic Gardens, Seattle. $1021 \mathrm{pp}$.

Mancini, M.V., M.M. Páez, A.R. Prieto, S. Stutz, M. Tonello \& I. Vilanova. 2005. Mid-Holocene climatic variability reconstruction from pollen records $\left(32^{\circ}-52^{\circ} \mathrm{S}\right.$, Argentina). Quaternary International 132: 47-59.

Markgraf, V., M. McGlone \& G. Hope. 1995. Neogene paleoenvironmental and paleoclimatic change in southern temperate ecosystems- a southern perspective. Trends in Ecology and Evolution 10: 143-147.

Marquínez, X., L.G. Lohmann, M.L.F. Salatino, A. Salatino \& F. GonZÁlez. 2009. Generic relationships and dating of lineages in Winteraceae based on nuclear (ITS) and plastid (rpS16 and psbA-trnH) sequence data. Molecular Phylogenetics and Evolution 53: 435-449.

Mathiasen, P. \& A.C. Premoli. 2010. Out in the cold: genetic variation of Nothofagus pumilio (Nothofagaceae) provides evidence for latitudinally distinct evolutionary histories in austral South America. Molecular Ecology 19: 371-385.

Morales, J.M., M. Sirombra \& A.D. Brown. 1995. Riqueza de árboles en las yungas argentinas. En: A.D. Brown \& H.R. Grau (eds.), Investigación, conservación y desarrollo en selvas subtropicales de montañas. pp. 163-174. Proyecto de desarrollo agroforestal, LIEY, Tucumán, Argentina.

Moreira-Muñoz, A. 2007. The Austral floristic realm revisited. Journal of Biogeography 34: 1649-1660.

Navarro G. \& W. Ferreira. 2004. Zonas de vegetación potencial de Bolivia: una base para el análisis de vacíos en conservación. Revista Boliviana de Ecología 15: 1-40.

Ortiz-Jaureguizar, E. \& G.A. Cladera. 2006. Paleoenvironmental evolution of southern South America during the Cenozoic. Journal of Arid Environments 66: 498-532.

Panero, J.L. \& V.A. FunK. 2008. The value of sampling anomalous taxa in phylogenetic studies: Major clades of the Asteraceae revealed. Molecular Phylogenetics and Evolution 47: 757782.

Pennington, R.T., M. Lavin, D.E. Prado, C.A. Pendry \& S.K. PELl. 2005. Climate change and speciation in neotropical seasonally dry forest plants. In: Y. Malhi \& O. Phillips (eds.). Tropical forest and global atmospheric change. pp. 199-214. Oxford University Press.

Placzek, C., J. Quade, J.L. Betancourt, P.J. Patchett, J.A. Rech, C. Latorre, A. Matmon, C. Holmgren \& N.B. English. 2009. Climate in the dry central Andes over geologic, millennial, and interanual timescales. Annals of Missouri Botanical Garden 96: 386-397.

Poole, I., D.J. Cantrill, P. Hayes \& J. Francis. 2000. The fossil record of Cunoniaceae: new evidence from Late Cretaceous wood of Antarctica? Review of Palaeobotany and Palynology 111: 127-144.

Poole, I., A.M.W. Mennega \& D.J. Cantrill. 2003. Valdivian ecosystems in the Late Cretaceous and Early Tertiary of Antarctica: further evidence from myrtaceous and eucryphiaceous fossil wood. Review of Palaeobotany and Palynology 124: 9-27.

Prámparo, M.B., M. Quattrocchio, M.A. Gandolfo, M.C. ZamaloA \& E. Romero. 2007. Historia evolutiva de las angiospermas (Cretácico-Paleógeno) en Argentina a través de los registros paleoflorísticos. Asociación Paleontológica Argentina. Publicación Especial Ameghiniana $50^{\circ}$ aniversario: $157-172$.

Quiroga, M.P. 2009. Contribución para la conservación de Podocarpaceae del cono sur a partir de patrones genéticos y biogeográficos. Tesis Doctoral. Universidad Nacional del Comahue, Centro Regional Bariloche. Argentina. 197 pp.

Quiroga, M.P. \& A.C. Premoli. 2007. Genetic patterns in Podocarpus parlatorei reveal the longterm persistence of coldtolerant elements in the southern Yungas. Journal of Biogeography 34: 447-455.

Ramos, V.A. \& S.M. KaY. 1992. Southern Patagonian plateau basalts and deformation: backarc testimony of ridge collisions. Tectonophysics 205: 261-282.

Ramos, V.A. 2005. Seismic ridge subduction and topography: Foreland deformation in the Patagonian Andes. Tectonophysics 399: 73-86.

Richardson, J., T. Pennington, T. Pennington \& P. Hollingsworth. 2001. Rapid diversification of a species-rich genus of Neotropical rain forest trees. Science 293: 2242-2245.

Romero, E.J. 1986. Paleogene phytogeography and climatology of South America. Annals of the Missouri Botanical Garden 73: 449-461.

Ruggiero, A. \& C. Ezcurra. 2003. Regiones y transiciones biogeográficas. En J.J. Morrone \& J. Llorente (eds.), 
Biogeografía en Latinoamérica 2. pp. 141-154. Facultad de Ciencias UNAM, México.

Simpson, B. 1983. A historical phytogeography of the high Andean flora. Revista Chilena de Historia Natural 56: 109-122.

Simpson, B. \& C. TodziA. 1990. Patterns and prosses in the development of the high Andean flora. American Journal of Botany 77: 1419-1432.

SoKAL, R. \& F. RohlF. 1981. Biometry. $2^{\text {nd }}$ ed. New York: Freeman. $859 \mathrm{pp}$.

Solbrig, O. 1978. The origin and floristic affinities of the South American temperate desert and semidesert regions. In: D.D. Goodall (ed.), Biodiversity and conservation of neotropical montane forest. pp. 7-49. The New York Botanical Garden. Bronx.

STERN, C.R. 2008. Holocene tephrochronology record of large explosive eruptions in the southernmost Patagonian Andes. Bulletin of Volcanology 70: 435-454.

Thomson, S.N., M.T. Brandon, P.W. Reiners, J.H. Tomkin, C. VÁsquez \& N.J. WiLSON. 2010. Glaciation as a destructive and constructive control on mountain building. Nature. In press, doi: 10.1038/nature09365.

Ulloa-UlloA, C. \& P. Møller-Jørgensen. 1993. Árboles y arbustos de los Andes del Ecuador. AAU Reports 30. Aarhus University Press, Aarhus. 264 pp.

Van der Hammen, T. \& H. Hooghiemstra. 2001. Historia y paleoecología de los bosques montanos andinos tropicales. En: Kappelle M. \& A.D. Brown (eds.), Bosques nublados del neotrópico. pp. 63-84. Editorial INBio. Costa Rica.

Villagrán, C. \& L. Hinojosa. 1997. Historia de los bosques del sur de Sudamérica, II análisis fitogeográfico. Revista Chilena de Historia Natural 70: 225-239.

Wiens, J.J. \& C.H. Graham. 2005. Niche conservatism: Integrating Evolution, Ecology, and Conservation Biology. Annual Review in Ecology, Evolution and Systematics. 36: 51939.

Wilf, P., N.R. Cuneo, K.R. Jonson, J.F. Hicks, S.L. Wing \& J.D. Obradovich. 2003. High plant diversity in Eocene South America: Evidence from Patagonia. Science 300: 122125.

Wilf, P., C. Labandeira, K. Jonson \& N. Cúneo. 2005. Richness of plant-insect associations in Eocene Patagonia: a legacy for South America biodiversity. Proocedings of the National Academy of Science of the United States of the America 102: 8944-8948.

Willis, K.J. \& J.M. MCELWAin. 2002. The evolution of plants. Oxford University Press.

Zachos, J., M. Pagani, L. Sloan, E. Thomas \& K. Billups. 2001. Trends, rhythms and aberrations in global climate $65 \mathrm{Ma}$ to Present. Science 292: 686-693.

Zuloaga, F.O., M. Belgrano \& O. Morrone. 2008. Catálogo de las Plantas Vasculares del Cono Sur (Argentina, sur de Brasil, Chile, Paraguay y Uruguay). Monographs in Systematic Botany 107: 1-3486. Missouri Botanical Garden Press, Saint Louis.

Material Suplementario: Lista de familias y géneros de especies con hábito arbóreo del bosque tucumano-boliviano (BTB), del bosque templado austral (BTA) y de distribución en ambos bosques (Común). Se indica para cada taxón el elemento biogeográfico. AU: Austral, AD: Andino, C: Cosmopolita, E: Endémico, H: Holártico, N: Neotropical, P: Pantropical, S/D: sin datos.

SuPPLEMENTARY MATERIAL: List of families and tree genera in the tucumano-boliviano forest (BTB) and the austral temperate forest (BTA), and the distribution in both forest (Común). Each biogeographical element is indicated for the taxon. AU: Austral, AD: Andean, C: Cosmopolitan, E: Endemic, H: Holartic, N: Neotropical, P: Pantropical, S / D: no data.

\begin{tabular}{|c|c|c|c|}
\hline Familia & Género & $\begin{array}{c}\text { Elemento biogeográfico } \\
\text { del género }\end{array}$ & $\begin{array}{c}\text { Distribución geográfica } \\
\text { del género }\end{array}$ \\
\hline Achatocarpaceae & Achatocarpus Triana & $\mathrm{N}$ & BTB \\
\hline Adoxaceae & Viburnum L. & $\mathrm{H}$ & ВТВ \\
\hline Aextoxicaceae & Aextoxicon Ruiz \& Pav. & $\mathrm{E}$ & BTA \\
\hline \multirow[t]{6}{*}{ Anacardiaceae } & Astronium Jacq. & $\mathrm{N}$ & BTB \\
\hline & Lithrea Hook. & $\mathrm{N}$ & Común \\
\hline & Loxopterygium Hook. f. & $\mathrm{N}$ & BTB \\
\hline & Mauria Kunth & $\mathrm{AD}$ & ВТВ \\
\hline & Schinopsis Engl. & $\mathrm{N}$ & ВTB \\
\hline & Schinus L. & $\mathrm{N}$ & Común \\
\hline Annonaceae & Rollinia A.St.-Hil. & $\mathrm{N}$ & BTB \\
\hline Apocynaceae & Rauvolfia Plum. ex L. & $\mathrm{P}$ & ВTB \\
\hline Aquifoliaceae & Ilex $\mathrm{L}$. & $\mathrm{C}$ & ВТВ \\
\hline \multirow[t]{2}{*}{ Araliaceae } & Oreopanax Decne. \& Planc. & $\mathrm{N}$ & ВТВ \\
\hline & Raukaua Seem. & $\mathrm{AU}$ & BTA \\
\hline Araucariaceae & Araucaria Juss. & $\mathrm{AU}$ & BTA \\
\hline Arecaceae & Acrocomia Mart. & $\mathrm{N}$ & BTB \\
\hline \multirow[t]{3}{*}{ Asteraceae } & Cnicothamnus Griseb. & $\mathrm{N}$ & ВТВ \\
\hline & Dasyphyllum Kunth. & $\mathrm{N}$ & Común \\
\hline & Eupatorium L. & $\mathrm{P}$ & BTB \\
\hline
\end{tabular}




\begin{tabular}{|c|c|c|c|}
\hline Familia & Género & $\begin{array}{c}\text { Elemento biogeográfico } \\
\text { del género }\end{array}$ & $\begin{array}{c}\text { Distribución geográfica } \\
\text { del género }\end{array}$ \\
\hline Betulaceae & Alnus Mill. & $\mathrm{H}$ & BTB \\
\hline \multirow[t]{4}{*}{ Bignoniaceae } & Handroanthus Mattos & $\mathrm{N}$ & BTB \\
\hline & Jacaranda Juss. & $\mathrm{N}$ & BTB \\
\hline & Tabebuia Gomez & $\mathrm{N}$ & ВТВ \\
\hline & Tecoma Juss. & $\mathrm{N}$ & ВTB \\
\hline \multirow[t]{4}{*}{ Bombacaceae } & Ceiba Mill. & $\mathrm{P}$ & ВТВ \\
\hline & Eriotheca Schott \& Endl & $\mathrm{N}$ & BTB \\
\hline & Pseudobombax Dungand & $\mathrm{N}$ & ВТВ \\
\hline & Tartagalia (A.Robyns) T.Mey. & $\mathrm{N}$ & ВTB \\
\hline \multirow{2}{*}{ Boraginaceae } & Cordia $\mathrm{L}$. & $\mathrm{P}$ & ВТВ \\
\hline & Saccellium Humb. \& Bonpl. & $\mathrm{N}$ & BTB \\
\hline Capparaceae & Capparis L. & $\mathrm{P}$ & ВТВ \\
\hline Cardiopteridaceae & Citronella D.Don & $\mathrm{N}$ & Común \\
\hline Caricaceae & Carica $\mathrm{L}$ & $\mathrm{N}$ & ВТВ \\
\hline \multirow[t]{3}{*}{ Celastraceae } & Maytenus Molina & $\mathrm{P}$ & Común \\
\hline & Plenckia Reissek & $\mathrm{N}$ & ВТВ \\
\hline & Schaefferia Jacq. & $\mathrm{N}$ & ВТВ \\
\hline \multirow[t]{3}{*}{ Celtidaceae } & Celtis L. & $\mathrm{H}$ & BTB \\
\hline & Trema Lour. & $\mathrm{P}$ & ВТВ \\
\hline & Clethra L. & $\mathrm{P}$ & ВТВ \\
\hline Clusiaceae & Clusia L. & $\mathrm{N}$ & ВТВ \\
\hline Combretaceae & Terminalia $\mathrm{L}$. & $\mathrm{P}$ & BTB \\
\hline \multirow[t]{2}{*}{ Cunoniaceae } & Caldcluvia D.Don & AU & BTA \\
\hline & Weinmannia $\mathrm{L}$ & AU & Común \\
\hline \multirow[t]{3}{*}{ Cupresaceae } & Pilgerodendron Florin & $\mathrm{E}$ & BTA \\
\hline & Austrocedrus Florin \& Boutelje & $\mathrm{E}$ & BTA \\
\hline & Fitzroya Hook.f. ex Lindl. & $\mathrm{E}$ & BTA \\
\hline \multirow[t]{3}{*}{ Elaeocarpaceae } & Aristotelia L’ Hér. & AU & BTA \\
\hline & Crinodendron Molina & $\mathrm{AU}$ & Común \\
\hline & Vallea Mutis ex L.f. & $\mathrm{N}$ & ВTB \\
\hline Ericaceae & Agarista D.Don & $\mathrm{N}$ & ВТВ \\
\hline Erythroxylaceae & Erythroxylum P.Browne & $\mathrm{N}$ & ВТВ \\
\hline Eucryphiaceae & Eucryphia Cav. & $\mathrm{AU}$ & BTA \\
\hline \multirow[t]{6}{*}{ Euphorbiaceae } & Cnidoscolus Pohl & $\mathrm{N}$ & ВТВ \\
\hline & Croton L. & $\mathrm{C}$ & ВТВ \\
\hline & Jatropha L. & $\mathrm{N}$ & ВТВ \\
\hline & Sapium Jacq. & $\mathrm{P}$ & BTB \\
\hline & Sebastiania Bertol. & $\mathrm{P}$ & ВТВ \\
\hline & Stillingia Bertol. & $\mathrm{N}$ & ВTB \\
\hline \multirow[t]{2}{*}{ Fabaceae Caesalpinioideae } & Bauhinia L. & $\mathrm{P}$ & ВТВ \\
\hline & Senna (Cav) H.S.Irwin \& Barneby & $\mathrm{P}$ & BTB \\
\hline \multirow[t]{8}{*}{ Fabaceae Mimosoideae } & Acacia Mill. & $\mathrm{P}$ & Común \\
\hline & Anadenanthera Speg. & $\mathrm{N}$ & BTB \\
\hline & Enterolobium Mart. & $\mathrm{N}$ & ВTB \\
\hline & Inga Mill. & $\mathrm{N}$ & ВТВ \\
\hline & Mimosa L. & $\mathrm{N}$ & ВТВ \\
\hline & Parapiptadenia Brenan & $\mathrm{N}$ & ВТВ \\
\hline & Piptadenia Benth. & $\mathrm{N}$ & ВТВ \\
\hline & Prosopis L. & $\mathrm{P}$ & ВТВ \\
\hline \multirow[t]{3}{*}{ Fabaceae Papilionoideae } & Amburana Schwacke \& Taub. & $\mathrm{N}$ & ВТВ \\
\hline & Erythrina L. & $\mathrm{P}$ & ВТВ \\
\hline & Lonchocarpus Kunth & $\mathrm{P}$ & BTB \\
\hline
\end{tabular}




\begin{tabular}{|c|c|c|c|}
\hline Familia & Género & $\begin{array}{c}\text { Elemento biogeográfico } \\
\text { del género }\end{array}$ & $\begin{array}{c}\text { Distribución geográfica } \\
\text { del género }\end{array}$ \\
\hline & Machaerium Pers & $\mathrm{N}$ & BTB \\
\hline & Myroxylon L.f. & $\mathrm{N}$ & ВTB \\
\hline & Sophora L. & $\mathrm{AU}$ & BTA \\
\hline & Tipuana Benth. & $\mathrm{N}$ & ВTB \\
\hline Fagaceae & Nothofagus Blume & $\mathrm{AU}$ & BTA \\
\hline Gomortegaceae & Gomortega Riuz \& Pav. & $\mathrm{E}$ & BTA \\
\hline Juglandaceae & Juglans L. & $\mathrm{H}$ & ВTB \\
\hline \multirow[t]{5}{*}{ Lauraceae } & Cinnamomum Schaeff. & $\mathrm{P}$ & ВTB \\
\hline & Cryptocarya R.Br. & $\mathrm{P}$ & BTA \\
\hline & Nectandra Rottb. & $\mathrm{N}$ & ВTB \\
\hline & Ocotea Aubl. & $\mathrm{N}$ & ВTB \\
\hline & Persea Mill. & $\mathrm{P}$ & Común \\
\hline Lythraceae & Adenaria Kunth & $\mathrm{N}$ & ВТВ \\
\hline Malpighiaceae & Ptilochaeta Turcz. & $\mathrm{N}$ & ВTB \\
\hline Melastomataceae & Miconia Ruiz \& Pav. & $\mathrm{N}$ & ВТВ \\
\hline \multirow[t]{2}{*}{ Meliaceae } & Cedrela P.Browne & $\mathrm{N}$ & BTB \\
\hline & Trichilia P.Browne & $\mathrm{P}$ & ВТВ \\
\hline \multirow[t]{3}{*}{ Monimiaceae } & Laurelia Juss. & $\mathrm{AU}$ & BTA \\
\hline & Laureliopsis Schodde & $\mathrm{E}$ & BTA \\
\hline & Peumus Molina & $\mathrm{AU}$ & BTA \\
\hline \multirow[t]{3}{*}{ Moraceae } & Ficus L. & $\mathrm{P}$ & ВTB \\
\hline & Maclura Nutt. & $\mathrm{P}$ & ВТВ \\
\hline & Morus L. & $\mathrm{C}$ & ВТВ \\
\hline Muntingiaceae & Muntingia L. & $\mathrm{N}$ & ВТВ \\
\hline Myricaceae & Morella Lour. & $\mathrm{P}$ & ВТВ \\
\hline Myrsinaceae & Myrsine L. & $\mathrm{N}$ & ВТВ \\
\hline \multirow[t]{14}{*}{ Myrtaceae } & Amomyrtella Kausel & $\mathrm{N}$ & BTB \\
\hline & Amomyrtus (Burret) D.Legrand \& Kausel & $\mathrm{E}$ & BTA \\
\hline & Blepharocalyx O.Berg & $\mathrm{N}$ & Común \\
\hline & Eugenia L. & $\mathrm{P}$ & ВТВ \\
\hline & Gomidesia O.Berg & $\mathrm{N}$ & ВTB \\
\hline & Legrandia Kausel & $\mathrm{E}$ & BTA \\
\hline & Luma A.Gray & $\mathrm{E}$ & BTA \\
\hline & Myrceugenia O.Berg & $\mathrm{N}$ & BTA \\
\hline & Myrcianthes O.Berg & $\mathrm{N}$ & ВТВ \\
\hline & Myrciaria O.Berg & $\mathrm{N}$ & ВТВ \\
\hline & Myrrhinium Schott & $\mathrm{N}$ & ВТВ \\
\hline & Paramyrciaria Kausel & $\mathrm{N}$ & ВТВ \\
\hline & Siphoneugena O.Berg & $\mathrm{N}$ & ВТВ \\
\hline & Tepualia Griseb. & $\mathrm{E}$ & BTA \\
\hline \multirow[t]{2}{*}{ Nyctaginaceae } & Bougainvillea Comm. ex Juss. & $\mathrm{N}$ & ВТВ \\
\hline & Pisonia L. & $\mathrm{N}$ & BTB \\
\hline Opiliaceae & Agonandra Miers ex Benth. & $\mathrm{N}$ & ВТВ \\
\hline Papaveraceae & Bocconia Plum. ex L. & $\mathrm{N}$ & ВTB \\
\hline Phyllanthaceae & Phyllanthus L. & $\mathrm{N}$ & ВТВ \\
\hline Piperaceae & Piper L. & $\mathrm{P}$ & ВТВ \\
\hline \multirow[t]{4}{*}{ Podocarpaceae } & Lepidothamnus Phil. & $\mathrm{AU}$ & BTA \\
\hline & Podocarpus L'Hér. ex Pers. & AU & Común \\
\hline & Prumnopitys Phil. & AU & Común \\
\hline & Saxegothaea Lindl. & $\mathrm{E}$ & BTA \\
\hline \multirow[t]{2}{*}{ Polygonaceae } & Coccoloba $\mathrm{L}$ & $\mathrm{N}$ & ВTB \\
\hline & Ruprechtia C.A.Mey. & $\mathrm{N}$ & BTB \\
\hline
\end{tabular}


Bosque templado austral vs. bosque tucumano-boliviano: QUIROGA, M.P.

\begin{tabular}{|c|c|c|c|}
\hline Familia & Género & $\begin{array}{c}\text { Elemento biogeográfico } \\
\text { del género }\end{array}$ & $\begin{array}{c}\text { Distribución geográfica } \\
\text { del género }\end{array}$ \\
\hline \multirow[t]{4}{*}{ Proteaceae } & Embothrium Forst. & $\mathrm{E}$ & BTA \\
\hline & Gevuina Molina & AU & BTA \\
\hline & Lomatia $\mathrm{R} . \mathrm{Br}$. & AU & BTA \\
\hline & Roupala Aubl. & $\mathrm{N}$ & ВТВ \\
\hline \multirow[t]{4}{*}{ Rhamnaceae } & Condalia Cav. & $\mathrm{N}$ & ВTB \\
\hline & Discaria Hook. & AU & BTA \\
\hline & Rhamnus L. & $\mathrm{H}$ & ВTB \\
\hline & Scutia (Comm. ex DC.) Borngn. & $\mathrm{N}$ & ВTB \\
\hline \multirow[t]{4}{*}{ Rosaceae } & Hesperomeles Lindl. & $\mathrm{N}$ & ВTB \\
\hline & Kageneckia Ruiz \& Pav. & $\mathrm{AD}$ & ВTB \\
\hline & Polylepis Ruiz \& Pav. & $\mathrm{AD}$ & ВТВ \\
\hline & Prunus L. & $\mathrm{H}$ & ВТВ \\
\hline \multirow[t]{3}{*}{ Rubiaceae } & Calycophyllum DC. & $\mathrm{N}$ & ВТВ \\
\hline & Coutarea Aubl. & $\mathrm{N}$ & ВТВ \\
\hline & Pogonopus Klotzsch & $\mathrm{N}$ & ВТВ \\
\hline Rutaceae & Zanthoxylum L. & $\mathrm{P}$ & ВТВ \\
\hline \multirow[t]{5}{*}{ Salicaceae } & Azara Ruiz \& Pav. & $\mathrm{AU}$ & BTA \\
\hline & Casearia Jacq. & $\mathrm{P}$ & BTB \\
\hline & Prockia P.Browne ex L. & $\mathrm{N}$ & BTB \\
\hline & Salix L. & $\mathrm{H}$ & Común \\
\hline & Xylosma G.Forst. & $\mathrm{P}$ & BTB \\
\hline Santalaceae & Acanthosyris Griseb. & $\mathrm{N}$ & ВTB \\
\hline \multirow[t]{4}{*}{ Sapindaceae } & Allophylus L. & $\mathrm{P}$ & ВТВ \\
\hline & Athyana Radlk. & $\mathrm{N}$ & ВTB \\
\hline & Cupania L. & $\mathrm{N}$ & ВТВ \\
\hline & Diatenopteryx Radlk. & $\mathrm{N}$ & ВTB \\
\hline \multirow[t]{2}{*}{ Sapotaceae } & Chrysophyllum L. & $\mathrm{P}$ & ВTB \\
\hline & Sideroxylon L. & $?$ & ВTB \\
\hline Simarubaceae & Alvaradoa Liebm. & $\mathrm{N}$ & ВТВ \\
\hline \multirow[t]{2}{*}{ Solanaceae } & Dunalia Kunth & $\mathrm{N}$ & ВТВ \\
\hline & Vassobia Rusby & $\mathrm{N}$ & ВТВ \\
\hline Styracaceae & Styrax L. & $\mathrm{H}$ & ВТВ \\
\hline Symplocaceae & Symplocos Jacq. & $\mathrm{N}$ & ВТВ \\
\hline \multirow[t]{2}{*}{ Theaceae } & Freziera Sw. ex Willd. & $\mathrm{N}$ & ВТВ \\
\hline & Ternstroemia L.f. & $\mathrm{P}$ & ВТВ \\
\hline \multirow[t]{2}{*}{ Tiliaceae } & Heliocarpus L. & $\mathrm{N}$ & ВТВ \\
\hline & Luehea Willd. & $\mathrm{N}$ & ВТВ \\
\hline Ulmaceae & Phyllostylon Capan. ex Benth. \& Hook.f. & $\mathrm{N}$ & ВТВ \\
\hline \multirow[t]{3}{*}{ Urticaceae } & Boehmeria Jacq. & $?$ & ВTB \\
\hline & Myriocarpa Benth. & $\mathrm{N}$ & BTB \\
\hline & Urera Gaudich. & $\mathrm{P}$ & BTB \\
\hline \multirow[t]{2}{*}{ Verbenaceae } & Citharexylum L. & $\mathrm{N}$ & ВТВ \\
\hline & Duranta L. & $\mathrm{N}$ & BTB \\
\hline Winteraceae & Drimys Forst. & $\mathrm{AU}$ & BTA \\
\hline
\end{tabular}

Recibido: 27.05.10

Aceptado: 13.08 .10 